\title{
Infrared and Chlorophyll Fluorescence Imaging Methods for Stress Evaluation
}

\author{
D. Michael Glenn ${ }^{1}$ \\ USDA-ARS-Appalachian Fruit Research Station, 2217 Wiltshire Road, Kearneysville, WV 25430 \\ Additional index words. thermography, differential thermal analysis, green fluorescent protein, reactive oxygen species, \\ charged coupled device camera
}

\begin{abstract}
Infrared and chlorophyll fluorescence imaging methods are useful techniques to evaluate environmental effects on plant performance. With the advent of digital imaging and advances in sensor technology, infrared (IR) thermography has become more accurate and less expensive. Modern IR cameras can resolve $0.5{ }^{\circ} \mathrm{C}$ temperature differences and researchgrade instruments can resolve $0.05^{\circ} \mathrm{C}$. This precision has allowed the physical processes of freezing and transpiration to be more accurately studied and modeled. Chlorophyll fluorescence imaging, although still an expensive technology, has also become sufficiently rugged to be useful in the field. The measurement of quantum efficiency, Fv/Fm, provides clear data on the effect of various environmental and biotic effects on the performance of photosynthesis in plants through the effect on photosystem II. Modern digital cameras with low signal-to-noise ratios can also image chlorophyll fluorescence using time lapse exposure. Peltier-cooled charge coupled device (CCD) cameras can measure the autoluminescence in stressed plants that is generated by reactive oxygen species. Advances in technology have reduced the cost and precision of imaging equipment to a point that they are more applicable tools to plant scientists.
\end{abstract}

Thermography, or IR imaging, measures the radiation bands from 9,000 to $14,000 \mathrm{~nm}$ using a sensor array in which each sensor is equitable with the pixel of a digital image. Each pixel in the digital image contains the emitted IR energy of the object that has been focused on the detection element. The numeric matrix of the digital image allows the surface temperature data to be calculated and manipulated in various ways through software based on the emissivity of the object. For example: 1) false color can be assigned to temperature ranges to aid in visualizing the temperature frequency distribution; 2) selected areas can have the temperature frequency distribution isolated for analysis or comparison with other selected objects; 3 ) narrow temperature bands can be selected for analysis; and 4) other depictions and analyses based on the researchers' needs. The scope and quality of IR cameras are diverse. Although the cost of IR cameras has decreased in the last decade, the primary concern when choosing an IR camera should be the accuracy and precision needed to accomplish the research objectives. The camera cost is driven by the accuracy and precision of the temperature measurement and can range from less than $\$ 1,000$ to $\$ 80,000+$. The more accurate and precise the temperature measurement, generally, the greater the cost of the camera and software. Cameras with absolute accuracy of $\pm 1{ }^{\circ} \mathrm{C}$ and precision of $0.05{ }^{\circ} \mathrm{C}$ are available and sufficiently rugged for field use. The primary advantage of thermography over thermocou-

Received for publication 2 Dec. 2011. Accepted for publication 10 Jan. 2012.

This paper was part of the colloquium, "Emerging Techniques to Evaluate and Mitigate Crop Environmental Stress in a Changing Climate" held 28 Sept. 2011 at the ASHS Conference, Waikoloa, HI, and sponsored by the Environmental Stress Physiology (STRS) Working Group.

${ }^{1}$ To whom reprint requests should be addressed; e-mail michael.glenn@ars.usda.gov. ple psychrometry is the ability to image objects over time while they move and with no physical contact and the ability to monitor large contiguous areas rather than point sources. Aside from cost, the two primary limitations to the value of thermography are 1) the accurate measurement of the target object emissivity; and 2) its ability to measure only one subject at a time. The object's emissivity is a required value for accurate calculation of surface temperature. Fortunately, plants in general have equivalent emissivity ( 0.95 to 0.98 ) and the measurement of emissivity is a simple matter. Thermography has led to a greater understanding of freezing (Wisniewski et al., 2008, 2009), heat stress (Chaerle et al., 2005), disease (Lenthe et al., 2007; Nilsson, 1995), insect detection (Nabity et al., 2009), and water use (Möller et al., 2007) as indicated in these recent, key reviews.

Recently, the concept of differential thermal analysis has further improved the information gained from IR images (Hacker et al., 2008). Although the hardware has not changed, a new image processing strategy has provided a refinement of the images. Basically, when a series of IR images are collected and an event or treatment is initiated, the image at the moment of initiation is used as a baseline image. The pixel values of the baseline image are subtracted from the pixel values from each subsequent image; as a result, pixels in which there is no change will remain dark or black in subsequent processed images, whereas those pixels representing some change in the surface temperature of the object will be gray to white. This analysis allows a greater understanding of the physical processes by providing a time lapse visualization.

Chlorophyll fluorescence and the photochemistry of photosystem II (PSII) can be imaged using cameras, sophisticated data capture techniques, and synchronized light sources (Baker, 2008). Both baseline chlorophyll fluorescence and the Fv/Fm can be imaged and used to understand the role of PSII in various physiological processes such as water stress, heat stress (Baker and Rosenqvist, 2004), and pest damage (Aldea et al., 2005; Berger et al., 2007). The value of imaging over simply measuring the photochemistry reaction from light stimulation for a designated area is that spatial variation is captured with extremely fine spatial resolution (less than $1 \mathrm{~mm}$ ). Similar to differential thermal analysis, differential chlorophyll fluorescence analysis can be performed (Hacker et al., 2008). When a series of Fo chlorophyll fluorescence images are collected and an event or treatment is initiated, the image at the moment of initiation is used as a baseline image. This image is "subtracted" from each subsequent image and so each subsequent image presents only the pixels/areas that are changing. This provides increased visual refinement of the images and understanding of the process.

The use of green fluorescent protein (GFP) expression and its imaging within cells has greatly increased knowledge concerning the organization of cellular activities (Hanson and Kohler, 2001) and signal transduction (Ehrhardt, 2003; Murphy et al., 2001). The conventional use of GFP imagery is based on still images or sequences of images under ultraviolet illumination. GFP will fluoresce under black light (peak of $370 \mathrm{~nm}$ ) in addition to broad-spectrum ultraviolet lamps. Using a black light on living plant tissue reduces possible ultraviolet cellular damage compared with a broad-spectrum ultraviolet source and provides long-term excitation of GFP reactions without damage to living tissue for time lapse photography. Examples of time lapse photography of GFP-induced fluorescence from viral infection are found in Manfre et al. (2011) (<http://apsjournals. apsnet.org/doi/pdfplus/10.1094/MPMI-0810-0191>, <http://apsjournals.apsnet.org/doi/ suppl/10.1094/MPMI-08-10-0191>). In these studies, white light and black light illumination were synchronized to alternately go off 
and on every 30 min with a 30 -s period of black light illumination of the plants allowing for a 25-s time exposure of the plants. Visualization of the infection process not only provides data on the sites and rates of infection but also integrates the plant's overall reaction, which includes leaf wilting.

Imaging oxidative stress responses in plants through various dyes are well established (Fryer et al., 2002) despite the short lifespan of the reactive oxygen species (ROS). Real-time imaging of ROS production generated from mechanical damage without dyes has been documented by Flor-Henry et al. (2004) and Havaux et al. (2006) using cooled CCD cameras. CCD cameras have a low signalto-noise ratio $(\mathrm{S} / \mathrm{N})$ and the cooling of the camera sensor further reduces the $\mathrm{S} / \mathrm{N}$. There are two approaches to cooling CCD cameras: 1) liquid nitrogen; and 2) thermoelectric cooling using the Peltier effect. The method and effectiveness of cooling not only reduce the $\mathrm{S} / \mathrm{N}$, but also raise the cost of the camera to the $\$ 40,000$ to $\$ 70,000+$ range. Various techniques can also improve the quantum capture efficiency of the camera. Electron multiplied CCD and intensified CCD (ICCD) cameras have efficiencies of $95 \%+$ and can image single photon emission. The ICCD camera is also called a gateable CCD camera and can achieve shutter speeds of picoseconds. Because ROS synthesis produces photon emissions (Havaux et al., 2006), advancing $\mathrm{CCD}$ technology can be expected to provide new tools to image this process. Because the chlorophyll molecule fluoresces in light and has residual fluorescence in the dark for several minutes (Flor-Henry et al., 2004; Havaux et al., 2006), this background fluorescence must be removed from images to visualize only ROS emissions. Modern CCD software and astronomy software (Parker, 1991) provide straightforward techniques to subtract a baseline image from subsequent images, similar to Hacker et al. (2008).
In summary, imaging of plant physiological processes is a new tool for plant biology research. It is providing a refinement of existing technology and adding new technology to answer/image questions previously impossible.

\section{Literature Cited}

Aldea, M., J.G. Hamilton, J.P. Resti, A.R. Zongerl, M.R. Berenbaum, and E.H. DeLucia. 2005. Indirect effects of insect herbivory on leaf gas exchange in soybean. Plant Cell Environ. 28:402-411.

Baker, N.R. 2008. Chlorophyll fluorescence: A probe of photosynthesis in vivo. Annu. Rev. Plant Biol. 59:89-113.

Baker, N.R. and E. Rosenqvist. 2004. Applications of chlorophyll fluorescence can improve crop production strategies: An examination of future possibilities. J. Exp. Bot. 55:1607-1621.

Berger, S., Z. Benediktyova, K. Matous, K. Bonfig., M.J. Mueller, L. Nedbal, and T. Roitsch. 2007. Visualization of dynamics of plant-pathogen interaction by novel combination of chlorophyll fluorescence imaging and statistical analysis: Differential effects of virulent and avirulent strains of $P$. syringae and of oxylipins on A. thaliana. J. Exp. Bot. 58:797-806.

Chaerle, L., N. Saibo, and D. Van Der Straeten. 2005. Tuning the pores: Towards engineering plants for improved water use efficiency. Trends Biotechnol. 23:308-315.

Ehrhardt, D. 2003. GFP technology for live cell imaging. Current Opinion Plant Biol. 6:622-628.

Flor-Henry, M.T., C. McCabe, G.L. de Bruxelles, and M.R. Roberts. 2004. Use of a highly sensitive two-dimensional luminescence imaging system to monitor endogenous bioluminescence in plant leaves. BMC Plant Biol. 4:19.

Fryer, M.J., K. Oxborough, P.M. Mullineaux, and N.R. Baker. 2002. Imaging of photo-oxidative stress responses in leaves. J. Exp. Bot. 53:1249-1254.

Hacker, J., J.P. Spindelböck, and G. Neuner. 2008. Mesophyll freezing and effects of freeze dehydration visualized by simultaneous measurement of IDTA and differential imaging chlorophyll fluorescence. Plant Cell Environ. 31:1725-1733.

Hanson, M.R. and R.H. Kohler. 2001. GFP imaging: Methodology and application to investigate cellular compartmentation in plants. J. Exp. Bot. 52:529-539.

Havaux, M., C.B. Triantaphylides, and B. Genty. 2006. Autoluminescence imaging: A noninvasive tool for mapping oxidative stress. Trends Plant Sci. 11:480-484.

Lenthe, J.H., E.C. Oerke, and H.W. Dehne. 2007. Digital infrared thermography for monitoring canopy health of wheat. Precision Agr. 8:1526.

Manfre, A., M. Glenn, A. Nuñez, R.A. Moreau, and C. Dardick. 2011. Light quantity and photosystem function mediate host susceptibility to Turnip mosaic virus via a salicylic acidindependent mechanism. Mol. Plant Microbe Interact. 24:315-327.

Möller, M., V. Alchanatis, Y. Cohen, M. Meron, J. Tsipris, A. Naor, V. Ostrovsky, M. Sprintsin, and S. Cohen. 2007. Use of thermal and visible imagery for estimating crop water status of irrigated grapevine. J. Exp. Bot. 58:827-838.

Murphy, A.M., A. Gilliland, C.E. Wong, J. West, D.P. Singh, and J.P. Carr. 2001. Signal transduction in resistance to plant viruses. Eur. J. Plant Pathol. 107:121-128.

Nabity, P.D., J.A. Zavala, and E.H. DeLucia. 2009. Indirect suppression of photosynthesis on individual leaves by arthropod herbivory. Ann. Bot. 103:655-663.

Nilsson, H. 1995. Remote sensing and image analysis in plant pathology. Annu. Rev. Phytopathol. 33:489-528.

Parker, J.W. 1991. Measuring the direct sky brightness on CCD images. Astronomical Soc. Pacific 103:243-252.

Wisniewski, M., D.M. Glenn, and M.P. Fuller. 2008. Using infrared thermography to study freezing in plants. HortScience 43:16481651.

Wisniewski, M., L.V. Gusta, M.P. Fuller, and D. Karlson. 2009. Ice nucleation, propagation and deep supercooling: The lost tribes of freezing studies, p. 1-11. In: L.V. Gusta, M.E. Wisniewski, and K.K. Tanino (eds.). Plant cold hardiness: From the laboratory to the field. CABI, Cambridge, MA. 